\title{
Head-up display may facilitate safe keyhole surgery for cerebral aneurysm clipping
}

\author{
Terushige Toyooka, MD, PhD, Naoki Otani, MD, PhD, Kojiro Wada, MD, PhD, \\ Arata Tomiyama, MD, PhD, Satoru Takeuchi, MD, PhD, Kazuya Fujii, MD, Kosuke Kumagai, MD, \\ Takashi Fujii, MD, and Kentaro Mori, MD, PhD
}

Department of Neurosurgery, National Defense Medical College, Tokorozawa, Saitama, Japan

\begin{abstract}
OBJECTIVE The head-up display (HUD) is a modern technology that projects images or numeric information directly into the observer's sight line. Surgeons will no longer need to look away from the surgical view using the HUD system to confirm the preoperative or navigation image. The present study investigated the usefulness of the HUD system for performing cerebral aneurysm clipping surgeries.

METHODS Thirty-five patients underwent clipping surgery, including 20 keyhole surgeries for unruptured cerebral aneurysm, using the HUD system. Image information of structures such as the skull, cerebral vasculature, and aneurysm was integrated by the navigation software and linked with the positional coordinates of the microscope field of view. "Image injection" allowed visualization of the main structures that were concurrently tracked by the navigation image, and "closed shutter" switched the microscope field of view and the pointer image of the 3D brain image.
\end{abstract}

RESULTS The HUD system was effective for estimating the location and 3D anatomy of the aneurysm before craniotomy or dural opening in most patients. Scheduled keyhole minicraniotomy and opening of the sylvian fissure or partial rectal gyrus resection were performed on the optimized location with a minimum size in 20 patients.

CONCLUSIONS The HUD images superimposed on the microscope field of view were remarkably useful for less invasive and more safe aneurysm clipping and, in particular, keyhole clipping.

https://thejns.org/doi/abs/10.3171/2017.5.JNS162692

KEY WORDS head-up display; keyhole surgery; cerebral aneurysm; clipping; vascular disorders

$\mathrm{O}$ PEN clipping surgery to treat cerebral aneurysms requires improvement in the safety of the procedures. In particular, localization and exposure of the aneurysm depends on the anatomical knowledge and expertise of the surgeon. However, even very experienced neurosurgeons sometimes experience difficulty in localizing the aneurysm and may damage the brain with unneeded dissection in certain cases, such as aneurysms in the distal middle cerebral artery (MCA) or posterior cerebral artery and abnormal arterial displacement due to thick hematoma. Keyhole surgery requires precise placement of the minicraniotomy at the point of the sylvian fissure opening for MCA aneurysms or rectal gyrus resection for anterior communicating artery (ACoA) aneurysms. Image-guided surgery using a computer-assisted navigation system has been extremely useful for treating various deeply located pathologies in daily neurosurgical practice.
However, the conventional navigation system monitor is remote from the operative field, which requires the surgeon to look away in order to check the monitor.

The head-up display (HUD) system is designed to provide image information directly in the operator's line of sight. The HUD system was originally developed for military aircraft pilots to display generic information such as airspeed, altitude, horizon line, flight data, and target designation in the view line of the pilot. The great advantage of this system is that the user need not look away from the current view field. Consequently, HUD can allow the surgeon to observe the navigation image without looking away from the surgical field to the secondary display. Therefore, the HUD navigation system is considered to give better visual feedback regarding the operative field. We recently started to use the HUD navigation system during aneurysm clipping surgeries at our institution.

ABBREVIATIONS $\mathrm{ACOA}=$ anterior communicating artery; $\mathrm{HUD}=$ head-up display; $I Q R=$ interquartile range; $M C A=$ middle cerebral artery .

SUBMITTED October 24, 2016. ACCEPTED May 15, 2017.

INCLUDE WHEN CITING Published online December 1, 2017; DOI: 10.3171/2017.5.JNS162692. 
Here we describe the use of the HUD navigation system for cerebral aneurysm clipping as well as our initial clinical experiences and assesses the feasibility of this technique in performing keyhole clipping surgeries.

\section{Methods}

This study included 35 patients with unruptured cerebral aneurysms who underwent open clipping surgery performed with the aid of HUD navigation systems at the National Defense Medical College Hospital between September 2014 and May 2016. For comparison, a control group of 41 patients who had undergone the same treatment before the induction of the HUD systems, between October 2012 and August 2014, were also analyzed. These cases were consecutive; large or giant aneurysms and complicated cases requiring skull base techniques were excluded. Clinical data were obtained retrospectively from the medical charts and neuroimages (Table 1). Fifteen patients in the HUD group and 20 patients in the control group were treated using standard frontotemporal craniotomy, and 20 patients in the HUD group and 21 patients in the control group were treated via keyhole minicraniotomy. The surgical procedures for keyhole clipping were as follows. Preoperative planning for keyhole surgery was mainly based on 3D CT angiography. A specific method based on surgical simulation using 3D images obtained in individual patients was applied to allow safe aneurysm clipping using computer simulation of the keyhole minicraniotomy. ${ }^{4}$ Pterional minicraniotomy via an outer canthal skin incision was adopted for clipping unruptured MCA aneurysms. ${ }^{5}$ Lateral supraorbital minicraniotomy via an eyebrow skin incision was adopted for clipping unruptured internal carotid artery or ACoA aneurysms. ${ }^{6}$

\section{HUD Systems}

Figure 1 shows the flowchart of the procedures performed using the HUD system. Image information acquired from CT or MRI is processed as layered data using the navigation software (iPlan Workstation, Brainlab) and transferred to the navigation devices (Brainlab). The positional information of the operating microscope (OPMI Pentero, Carl Zeiss) and the patient's head are precisely tracked with the navigation system and infrared camera. Segmented object information is integrated as HUD information on the microscope field of view. The HUD system has 2 viewing functions on the microscope field of view: image injection and closed shutter (Fig. 2). Image injection enables us to estimate deep anatomical information tracked by the segmented object image displayed on the microscope field of view in real time. Closed shutter enables us to assess 3D anatomy by switching from the operative field to the navigation image without turning our eyes away from the microscope field of view.

\section{Statistical Analysis}

Complications and outcomes were compared between the HUD and control groups. Craniotomy size and operative time for keyhole craniotomy were also analyzed as additional parameters. The t-test was used for comparison of the HUD and control groups; $p<0.05$ was considered-
TABLE 1. Clinical characteristics of patients who underwent clipping surgery for cerebral aneurysms before and after introduction of the HUD system

\begin{tabular}{lcc}
\hline \multicolumn{1}{c}{ Characteristic } & Control Group & HUD Group \\
\hline Treatment date & $\begin{array}{c}\text { October 2012 } \\
\text { to August 2014 }\end{array}$ & $\begin{array}{c}\text { September 2014 } \\
\text { to May 2016 }\end{array}$ \\
\hline No. of patients & 41 & 35 \\
\hline Sex, $\mathrm{n}$ & 15 & 11 \\
\hline Male & 26 & 24 \\
\hline Female & & \\
\hline Age, yrs & & \\
\hline Range & $44-77$ & \\
\hline Mean & 63.5 & 62.3 \\
\hline Craniotomy procedure, $\mathrm{n}$ & & 20 \\
\hline Keyhole & 21 & 15 \\
\hline $\begin{array}{l}\text { Standard } \\
\text { Location of aneurysm/keyhole, } \\
\mathrm{n}(\%)\end{array}$ & 20 & \\
\hline$\quad$ Distal anterior cerebral artery & $0(0)$ & $2(0)$ \\
\hline ACoA & $10(5)$ & $6(5)$ \\
\hline MCA & $24(11)$ & $21(12)$ \\
\hline Internal carotid artery & $7(5)$ & $6(3)$ \\
\hline
\end{tabular}

significant. The data are shown as the median with interquartile range (IQR).

\section{Results}

All 35 patients underwent clipping surgery for unruptured cerebral aneurysms and were discharged with no major complications and no permanent neurological deficits. Only 1 patient in the HUD group and 1 patient in the control group had postoperative events, which consisted of cerebral ischemia and hemorrhage, respectively, but without permanent symptoms. The operative time for keyhole surgery showed no significant difference between the HUD and control groups $(202 \pm 42$ minutes vs $192 \pm 41$ minutes, respectively). The characteristics of the keyhole surgery for the 20 patients who underwent aneurysm clipping were as follows: skin incision of $35-45 \mathrm{~mm}$, pterional craniotomy of $23 \mathrm{~mm}$ (IQR 22-25 mm) $\times 22 \mathrm{~mm}$ (IQR 20-23 mm), lateral supraorbital craniotomy of 28 $\mathrm{mm}$ (IQR 27-28.25 mm) $\times 21 \mathrm{~mm}$ (IQR 20-23 mm), and dural incision of $20 \mathrm{~mm}$ (IQR 17-21 mm) $\times 15 \mathrm{~mm}$ (IQR $15-16.5 \mathrm{~mm})$. Craniotomy size was significantly smaller in the HUD group than in the control group for the pterional keyhole, but not for the lateral supraorbital keyhole (Fig. 3).

\section{Illustrative Cases Case 1}

A patient with a right MCA aneurysm was treated via the right pterional keyhole approach using the HUD system (Fig. 4) (Videos 1 and 2).

VIDEO 1. Case 1. A 59-year-old female patient underwent clipping surgery via a left pterional keyhole craniotomy for a left MCA aneu- 


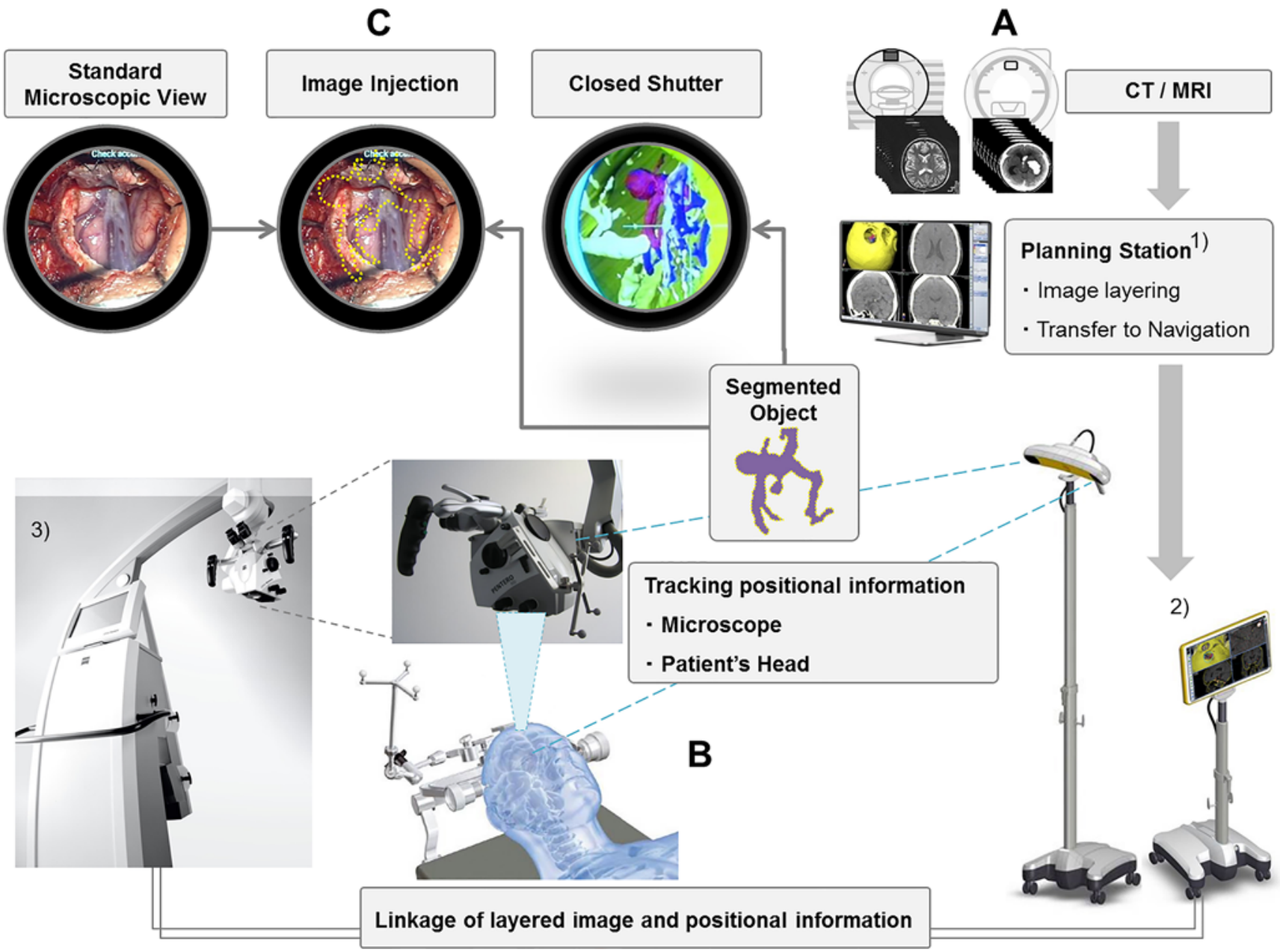

FIG 1. Flowchart of the procedures from registration of image data to linkage with the HUD system. A: Image information acquired by CT or MRI is processed as layered data using the navigation software (iPlan Workstation, Brainlab) (1) and transferred to the navigation devices (Brainlab) (2). B: Positional information of the operating microscope (OPMI Pentero) (3) and the patient's head are precisely tracked with the navigation system and infrared camera. Copyright Carl Zeiss Meditec, Inc. Published with permission. C: Segmented object information is integrated as HUD information on the microscope field of view. The HUD system has 2 viewing functions on the microscope field of view: image injection and closed shutter.

rysm with the aid of the HUD system. Using "image injection," the aneurysm location was estimated before the dural incision. Using "closed shutter," the 3D information of the aneurysm and affected arteries are provided. The dome of the aneurysm was estimated to be bedded under the temporal lobe. The aneurysm was completely exposed and clipped as expected. Copyright Department of Neurosurgery, National Defense Medical College. Published with permission. Click here to view.

VIDEO 2. Case 1. A 59-year-old female patient underwent clipping surgery via a right pterional keyhole craniotomy for a right MCA aneurysm with the aid of the HUD system. After the right pterional keyhole was made, HUD showed that the aneurysm was located more laterally. Therefore, the keyhole was extended $2 \mathrm{~mm}$ laterally using a high-speed drill. The aneurysm embedded in the temporal lobe was exposed and successfully clipped. This case shows HUD is useful for adjusting the location and size of the keyhole minicraniotomy during the operation. Copyright Department of Neurosurgery, National Defense Medical College. Published with permission. Click here to view.

The craniotomy, dural opening, and locations of specific structures were estimated on the HUD images before skin incision, which helped us to adjust the opening location or size. Craniotomy was extended by drilling to only the necessary size and direction. HUD information was useful for estimating the location of the aneurysm just beneath the superficial middle cerebral vein and minimizing arachnoid dissection to that necessary and sufficient for the clipping maneuver.

\section{Case 2}

A patient with an ACoA aneurysm was treated via the right supraorbital keyhole approach (Fig. 5). After opening the opticocarotid cistern via the subfrontal approach, the HUD system indicated that the aneurysm was hidden under the rectal gyrus. Minimum aspiration of the rectal gyrus was performed for the clipping maneuver based on the HUD information.

\section{Discussion}

The frameless neuronavigation system is mandatory for 


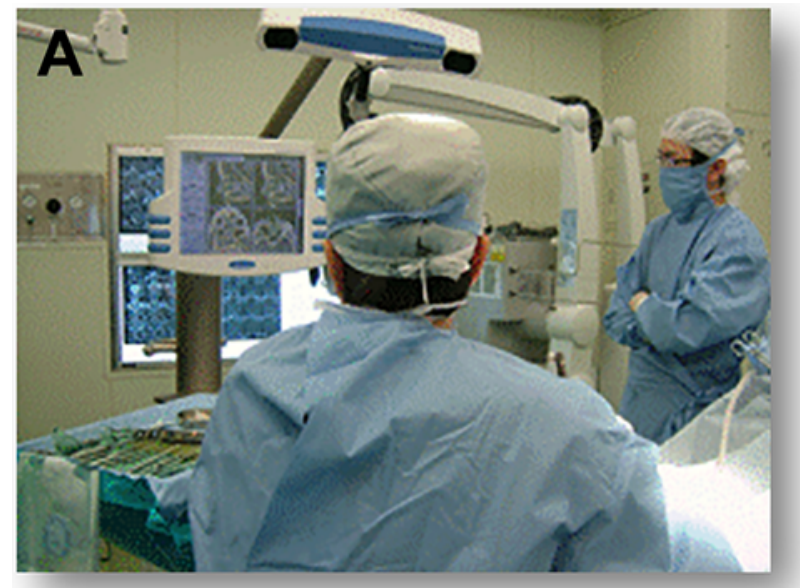

\section{Head-up display}
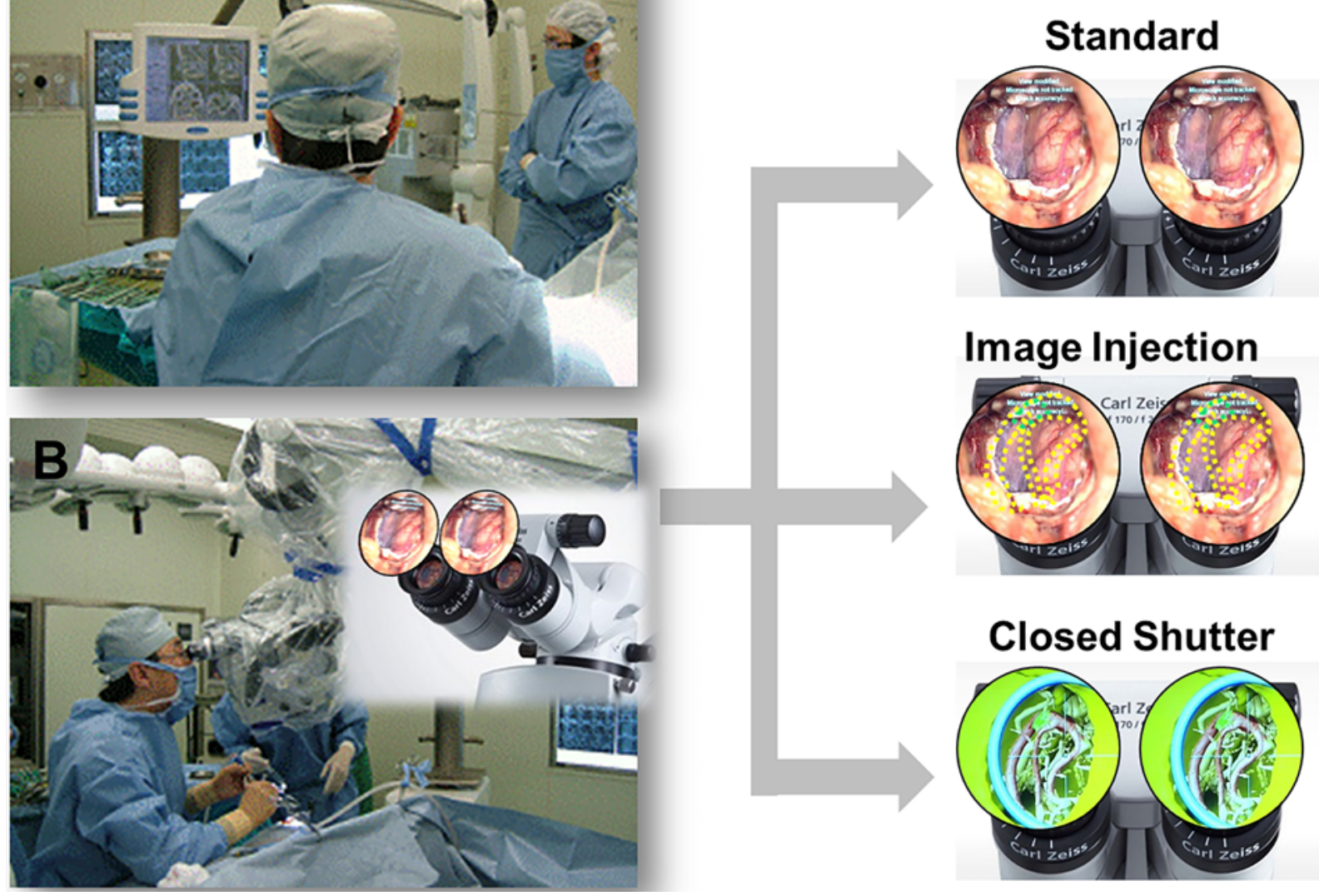

FIG 2. Intraoperative maneuver using the HUD functions. A: In standard neuronavigation, the surgeon confirms the structures on the navigation monitor by looking away from the operative field. B: Using HUD, the surgeon confirms the structures without turning away from the microscope field of view. C: Image injection enables us to estimate deep anatomical information tracked by the segmented object image displayed on the microscope field of view in real time. Closed shutter enables us to assess 3D anatomy by switching from the operative field to the navigation image.

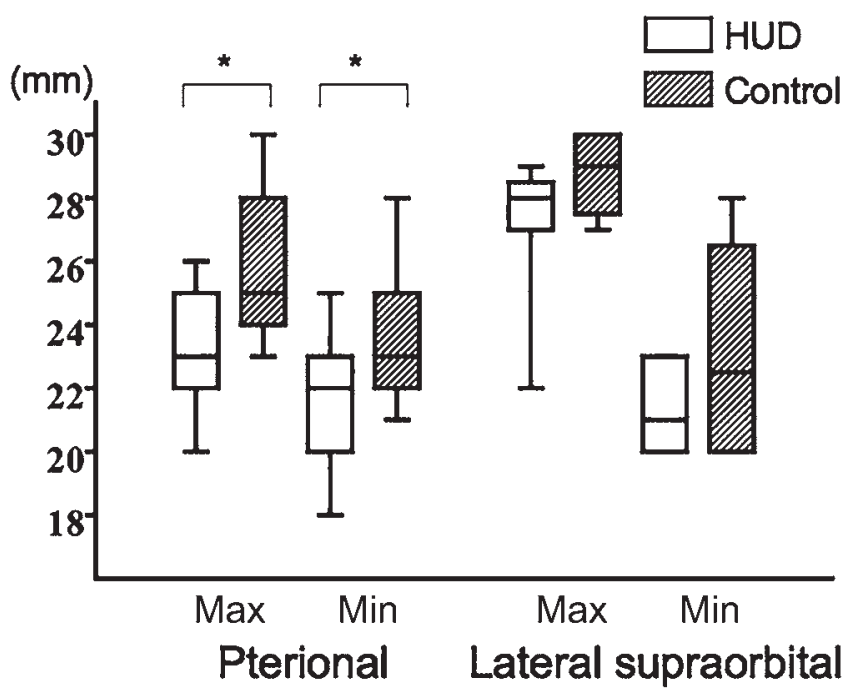

FIG 3. Craniotomy size in keyhole clipping surgeries for unruptured cerebral aneurysms. For these box-and-whiskers plots, the box indicates the IQR, the middle line indicates the median value, and the whiskers indicate the range. ${ }^{*} p<0.05$. routine neurosurgical operations such as brain tumor surgery, especially glioma surgery, but is less common in cerebrovascular surgery for various reasons. , $^{1,8}$ Navigation based on 3D digital subtraction angiography with or without other imaging modalities has been applied to open clipping surgery, but the main methods of data acquisition for neuronavigation are MRI and/or CT with lower sensitivity and spatial resolution for small aneurysms. ${ }^{11,12}$ Aneurysm clipping surgery is based on the so-called "cisternal approach" rather than the "trajectory approach"; thus surgeons tend to rely on anatomical knowledge and experience to localize the aneurysm and avoid looking away from the operative field to check the navigation image on a separate monitor. However, aneurysm localization is sometimes hindered by an uncommon aneurysm location, such as a distal MCA aneurysm or posterior cerebral artery aneurysm embedded in brain tissue, abnormally shifted vasculature due to hematoma, or restricted operative field for keyhole surgery. Therefore, navigation for open clipping surgery is considered to be useful in specific cases. ${ }^{2,7,9,10}$

The present study described our primary experience of performing aneurysm clipping surgery using an HUD 

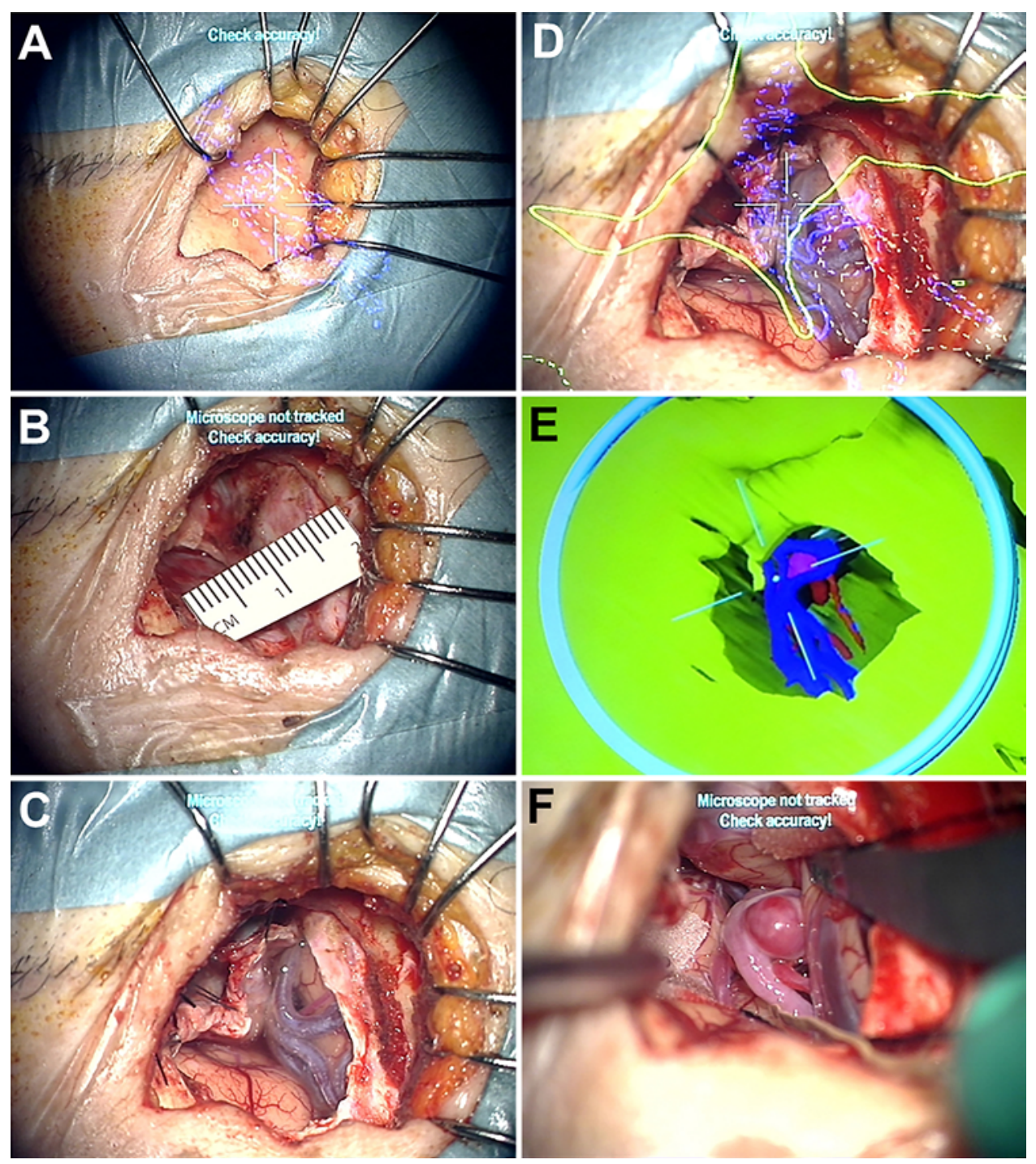

FIG 4. Case 1. Right MCA aneurysm treated via the right pterional keyhole approach. A: Before craniotomy, the location of specific structures such as the superficial middle cerebral vein, MCA, and aneurysm are estimated by image injection. B: Keyhole craniotomy was minimized with the aid of HUD. C: After semicircular dura incision, the running aspect of the superficial middle cerebral vein is confirmed to match the image as expected on the HUD before craniotomy. D: Image injection shows the 2D location of the aneurysm and the surrounding structures including the superficial middle cerebral vein. E: Closed shutter clarifies the location of the aneurysm just beneath the superficial middle cerebral vein with the 3D image. F: After minimization of the arachnoid incision based on the HUD information, the aneurysm is detected at the expected location under the superficial middle cerebral vein.

navigation system. The scheduled best location and size of the keyhole minicraniotomy were projected in the microscope field of view of the patient's face by HUD to facilitate the optimal skin incision. The scheduled keyhole minicraniotomy was also projected on the view of the exposed skull so precisely that the same bone window was the preoperatively determined target. The aneurysm was projected on the view of the exposed brain surface, which helped to minimize the sylvian fissure opening in cases of MCA aneurysms and rectal gyrus resection in cases of ACoA aneurysms. Although brain shift during intradural procedures remains a problem with navigation-guided surgery, the HUD system helped to localize and manipulate the aneurysm embedded in the brain tissue under the di- rect microscope view. At each key step, the HUD images superimposed in the microscope field of view helped the operator to precisely conduct the surgical manipulations and avoid unnecessary dissection without looking away from the operative field.

Unfortunately, the image quality on HUD is poorer than that on the original images because the image information acquired using 3D CT angiography is preoperatively compressed by the layering process of the navigation software to prevent intraoperative freezing of the navigation systems due to data overload. However, the present quality of 3D rendering was still useful in that the surgeon could confirm the angiographic 3D image superimposed on the surgical field without moving the line of sight from 

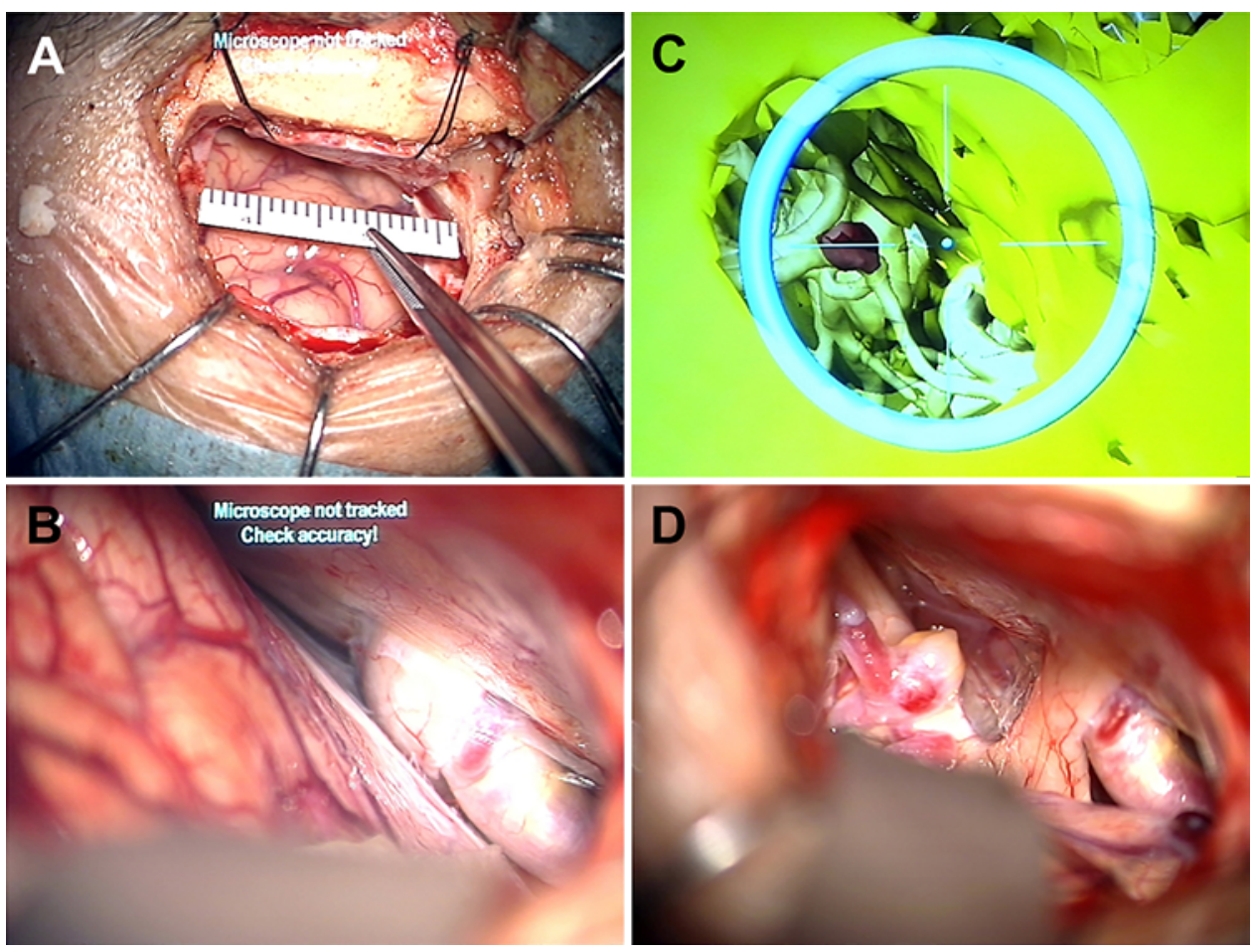

FIG 5. Case 2. ACoA aneurysm treated via keyhole craniotomy. A: Right supraorbital keyhole craniotomy with a 20-mm diameter. B: The opticocarotid cistern is opened via the subfrontal approach. C: Closed shutter shows the location of the aneurysm hidden under the rectal gyrus. D: After minimization of the aspiration of the rectal gyrus based on HUD information, the aneurysm is detected at the expected location.

the operating microscope. In the future, HUD information without deteriorated image quality might be achieved by improving both the software and hardware used in the navigation system.

The craniotomy size of the pterional keyhole was significantly smaller in the HUD group than in the control group, although that of the lateral supraorbital keyhole showed no significant difference. Minimizing the craniotomy was not our only objective, but the HUD was clearly useful for minimizing the craniotomy, or the opening site of the brain, before exposing the aneurysm. Although the HUD gave only an incremental improvement over standard navigation, the surgeons were given a sense of stability by the superimposition of the location or 3D structures of the aneurysm and the surrounding vessels on the surgical field view.

\section{Conclusions}

HUD images superimposed on the microscope field of view were remarkably useful for less invasive and safe aneurysm clipping and, in particular, keyhole clipping.

\section{References}

1. Barnett GH, Miller DW, Weisenberger J: Frameless stereotaxy with scalp-applied fiducial markers for brain biopsy procedures: experience in 218 cases. J Neurosurg 91:569-576, 1999

2. Lee SH, Bang JS: Distal middle cerebral artery M4 aneurysm surgery using navigation-CT angiography. J Korean Neurosurg Soc 42:478-480, 2007
3. Maesawa S, Fujii M, Nakahara N, Watanabe T, Saito K, Kajita Y, et al: Clinical indications for high-field $1.5 \mathrm{~T}$ intraoperative magnetic resonance imaging and neuro-navigation for neurosurgical procedures. Review of initial 100 cases.

Neurol Med Chir (Tokyo) 49:340-350, 2009

4. Mori K, Esaki T, Yamamoto T, Nakao Y: Individualized pterional keyhole clipping surgery based on a preoperative three-dimensional virtual osteotomy technique for unruptured middle cerebral artery aneurysm. Minim Invasive Neurosurg 54:207-213, 2011

5. Mori K, Osada H, Yamamoto T, Nakao Y, Maeda M: Pterional keyhole approach to middle cerebral artery aneurysms through an outer canthal skin incision. Minim Invasive Neurosurg 50:195-201, 2007

6. Mori K, Yamamoto T, Nakao Y, Oyama K, Esaki T, Watanabe M, et al: Lateral supraorbital keyhole approach to clip unruptured anterior communicating artery aneurysms. Minim Invasive Neurosurg 51:292-297, 2008

7. Raabe A, Beck J, Rohde S, Berkefeld J, Seifert V: Threedimensional rotational angiography guidance for aneurysm surgery. J Neurosurg 105:406-411, 2006

8. Roberts DW, Strohbehn JW, Hatch JF, Murray W, Kettenberger H: A frameless stereotaxic integration of computerized tomographic imaging and the operating microscope. $\mathbf{J}$ Neurosurg 65:545-549, 1986

9. Schmid-Elsaesser R, Muacevic A, Holtmannspötter M, Uhl E, Steiger HJ: Neuronavigation based on CT angiography for surgery of intracranial aneurysms: primary experience with unruptured aneurysms. Minim Invasive Neurosurg 46:269277,2003

10. Son YJ, Han DH, Kim JE: Image-guided surgery for treatment of unruptured middle cerebral artery aneurysms. Neurosurgery 61 (5 Suppl 2):266-272, 2007

11. Stidd DA, Wewel J, Ghods AJ, Munich S, Serici A, Keigher 
KM, et al: Frameless neuronavigation based only on 3D digital subtraction angiography using surface-based facial registration. J Neurosurg 121:745-750, 2014

12. Tee JW, Dally M, Madan A, Hwang P: Surgical treatment of poorly visualised and complex cerebrovascular lesions using pre-operative angiographic data as angiographic DynaCT datasets for frameless stereotactic navigation. Acta Neurochir (Wien) 154:1159-1167, 2012

\section{Disclosures}

The authors report no conflict of interest concerning the materials or methods used in this study or the findings specified in this paper.

\section{Author Contributions}

Conception and design: Mori. Acquisition of data: Toyooka, $\mathrm{K}$ Fujii, Kumagai, T Fujii, Mori. Analysis and interpretation of data: Toyooka, Otani, Wada, Tomiyama, Takeuchi, T Fujii, Mori. Drafting the article: Toyooka, T Fujii, Mori. Critically revising the article: Tomiyama, Kumagai, Mori. Reviewed submitted version of manuscript: Toyooka, Otani, Wada, Takeuchi, K Fujii, Mori. Approved the final version of the manuscript on behalf of all authors: Toyooka. Administrative/technical/material support: Otani, Wada, Tomiyama, Takeuchi, K Fujii, Kumagai, Mori. Study supervision: Mori.

\section{Supplemental Information \\ Videos}

Video 1. https://vimeo.com/225391838.

Video 2. https://vimeo.com/225391914.

\section{Correspondence}

Terushige Toyooka, Department of Neurosurgery, National Defense Medical College, 3-2 Namiki, Tokorozawa, Saitama 3598513, Japan. email: terutoy0807@gmail.com. 